2019 May;120:184-188.

doi: 10.1016/j.ijporl.2019.02.045. Epub 2019 Feb 27.

\title{
Comparison of distortion product otoacoustic emission (DPOAE) and automated auditory brainstem response (AABR) for neonatal hearing screening in a hospital with high delivery rate
}

Ling Xiu Ngui ${ }^{1}$, Ing Ping Tang 2, Narayanan Prepageran ${ }^{3}$, Zhun Wieng Lai ${ }^{4}$

Affiliations expand

- PMID: 30844634

- DOI: $10.1016 /$ j.ijporl.2019.02.045

Abstract

Introduction: Congenital hearing loss is one of the commonest congenital anomalies. Neonatal hearing screening aims to detect congenital hearing loss early and provide prompt intervention for better speech and language development. The two recommended methods for neonatal hearing screening are otoacoustic emission (OAE) and automated auditory brainstem response (AABR).

Objective: To study the effectiveness of distortion product otoacoustic emission (DPOAE) and automated auditory brainstem response (AABR) as first screening tool among non-risk newborns in a hospital with high delivery rate.

Method: A total of 722 non-risk newborns (1444 ears) were screened with both DPOAE and $A A B R$ prior to discharge within one month. Babies who failed $A A B R$ were rescreened with $A A B R \pm$ diagnostic auditory brainstem response tests within one month of age.

Results: The pass rate for AABR (67.9\%) was higher than DPOAE (50.1\%). Both DPOAE and $A A B R$ pass rates improved significantly with increasing age ( $p$-value $<0.001)$. The highest pass rate for both DPOAE and AABR were between the age of 36-48 h, 73.1\% and $84.2 \%$ respectively. The mean testing time for AABR (13.54 min \pm 7.47$)$ was significantly longer than DPOAE (3.52 min \pm 1.87 ), with a $p$-value of $<0.001$. 
Conclusions: $\mathrm{OAE}$ test is faster and easier than $A A B R$, but with higher false positive rate. The most ideal hearing screening protocol should be tailored according to different centre.

Keywords: Hearing loss; Infant; Neonatal screening; Newborn.

Copyright @ 2019 Elsevier B.V. All rights reserved. 